\title{
Investigation of a ceramic vane with a metal disk thermal and mechanical contact in a gas turbine impeller
}

\author{
S.V. Resnick ${ }^{\mathrm{a}}$, P.V. Prosuntsov and D.V. Sapronov \\ Bauman Moscow State Technical University, 105005 Moscow, Russia
}

\begin{abstract}
Promising directions of a new generation gas turbine engines development include using in gas turbines ceramic materials blades with high strength, thermal and chemical stability. One of the serious problems in developing such motors is insufficient knowledge of contact phenomena occurring in ceramic and metal details connection nodes. This work presents the numerical modeling results of thermal processes on ceramic and metal details rough boundaries. The investigation results are used in conducting experimental researches in conditions reproducing operating.
\end{abstract}

\section{Numerical modeling}

The purpose of this work - numerical modeling of thermal processes on ceramic and metal details rough boundaries.

The investigated object is the nitride ceramic OTM-914 blade and nickel alloy gas turbine disk contact area. At the simulation, the roughness data has been used, obtained on the basis of model profilograms materials primary analysis. As the main parameters there have been taken: the roughness maximum value $R_{\max }$, the average profile roughness step $S_{m i}$, the average distance between roughnesses $l_{m i}$ (Fig. 1), the roughnesses height in ten points $R_{Z}$, the profile filling coefficient $k_{p}$, and the ratio of actual to nominal contact area $F_{f} / F_{n}$.

Investigations have been carried out using a finite element contact area model.

Fictitious isothermal small thickness layers, endowed with infinitely high heat conductivity, have been introduced on the upper and lower model boundaries. The temperature $T_{1}$ has been applied to the nickel detail upper part, the temperature $T_{2}$ - to the ceramic detail lower part. The model upper part corresponded to the metal detail, and the lower part - to the ceramic. When considering radiative heat transfer in the gap, nickel alloy emissivity has been assumed of 0.09 , and silicon nitride - of 0.9 .

The simulation objective was to determine the ceramic and metal details contact layer thermal conductivity coefficient $\left(\lambda_{1}\right)$, which can be expressed as follows:

$$
\lambda_{l}=q_{t} \cdot \frac{\Delta}{\left(T_{1}-T_{2}\right)} .
$$

\footnotetext{
${ }^{a}$ Corresponding author: sreznik@bmstu.ru
}

This is an Open Access article distributed under the terms of the Creative Commons Attribution License 4.0, which permits unrestricted use, distribution, and reproduction in any medium, provided the original work is properly cited. 
EPJ Web of Conferences

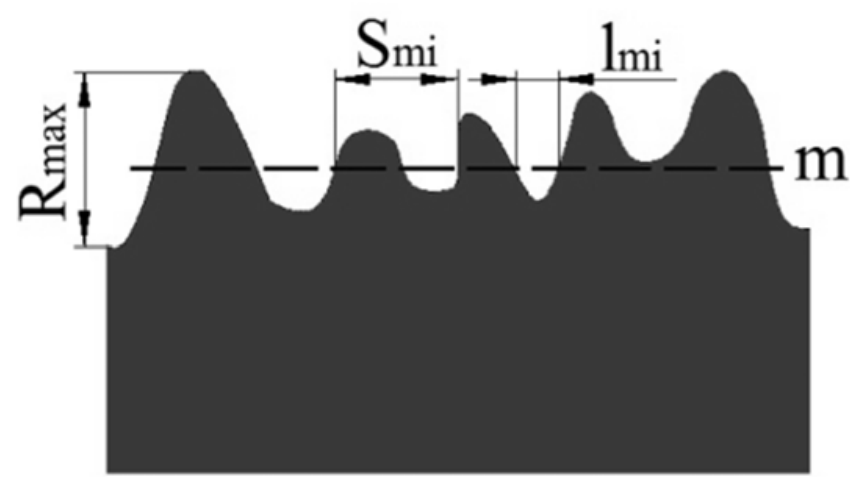

Figure 1. Real surface roughness profile.

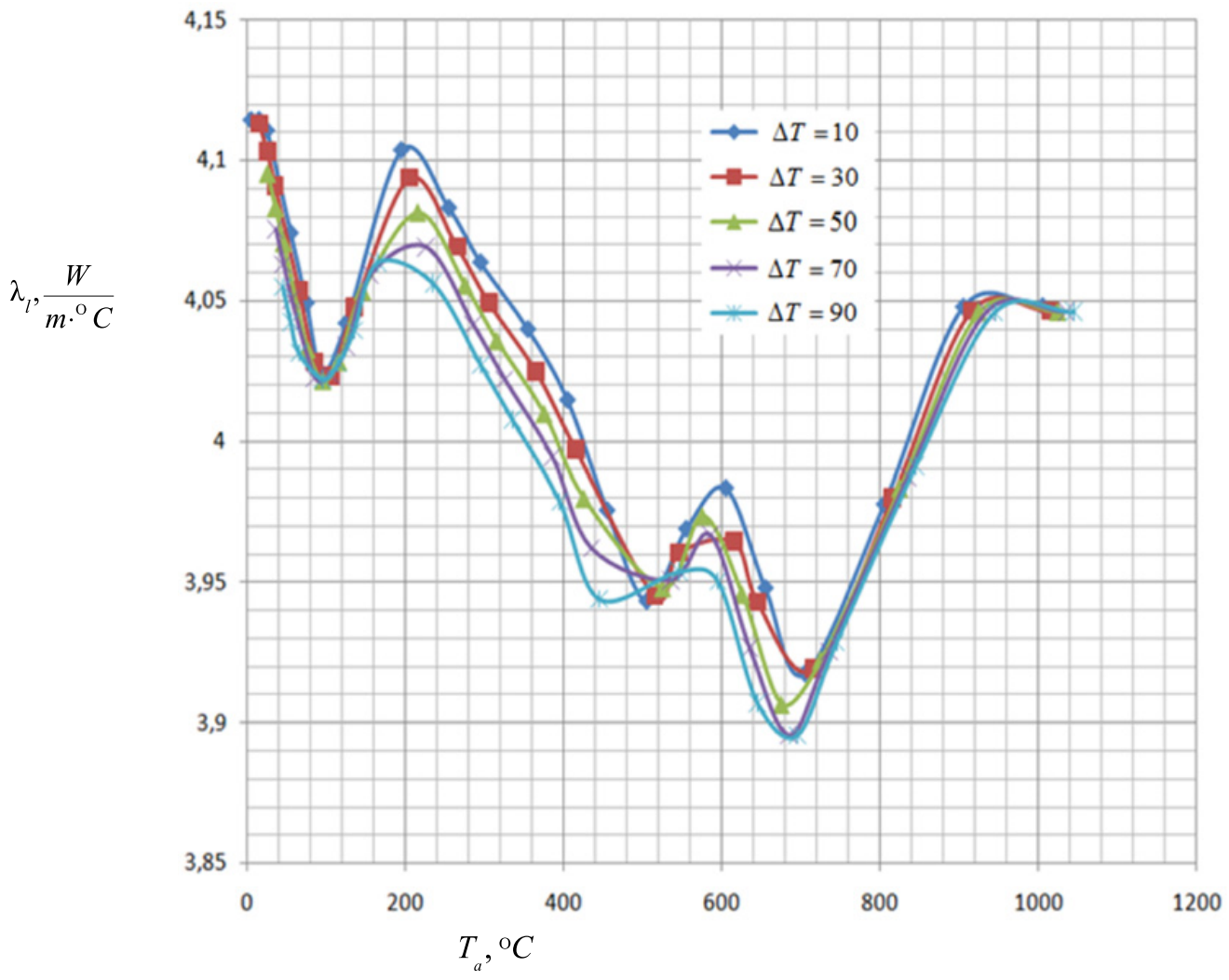

Figure 2. The dependence of the contact layer thermal conductivity coefficient on the average temperature in the contact zone $T_{a}$ at different $\Delta T_{c}$. 
Thermophysical Basis of Energy Technologies

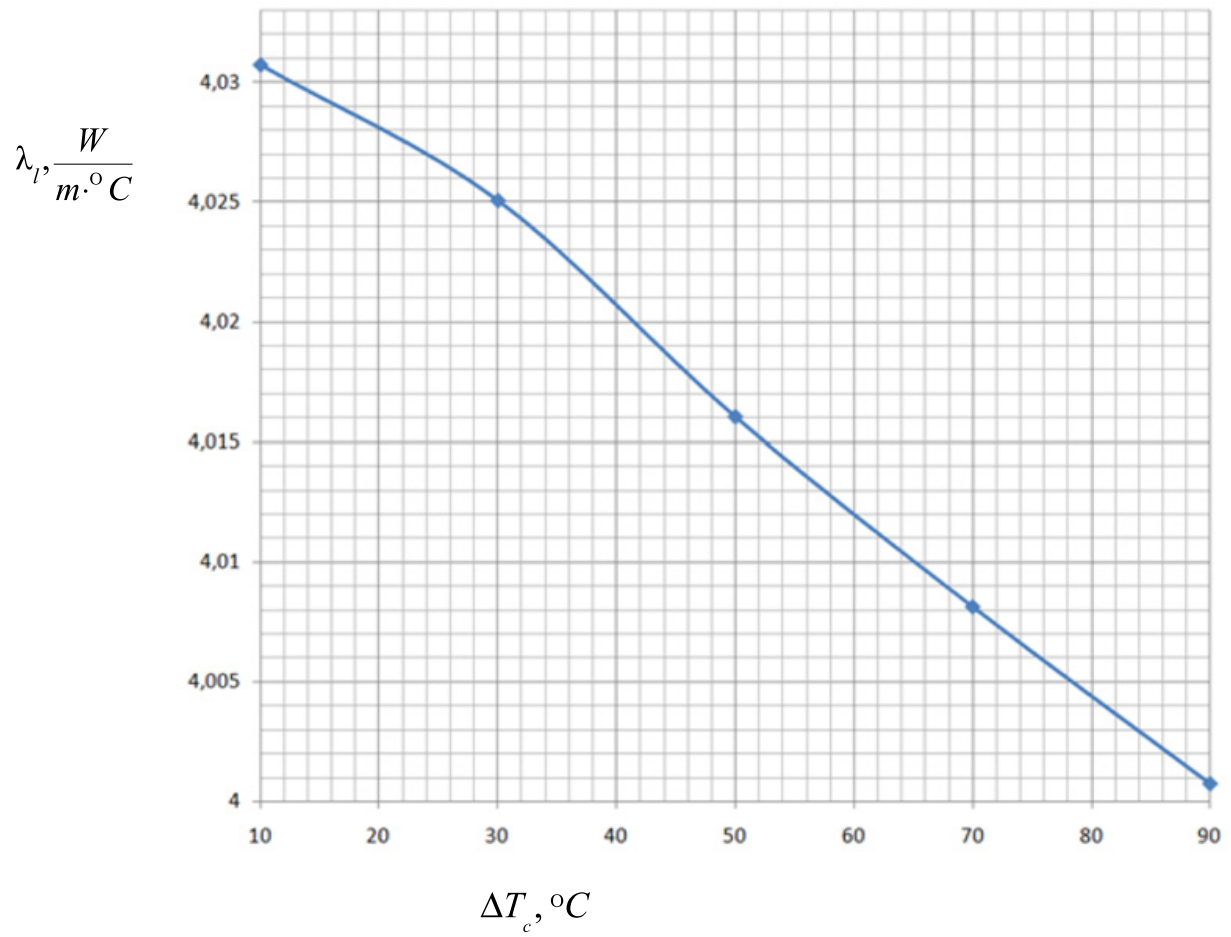

Figure 3. The dependence of the contact layer thermal conductivity coefficient on $\Delta T_{c}$.

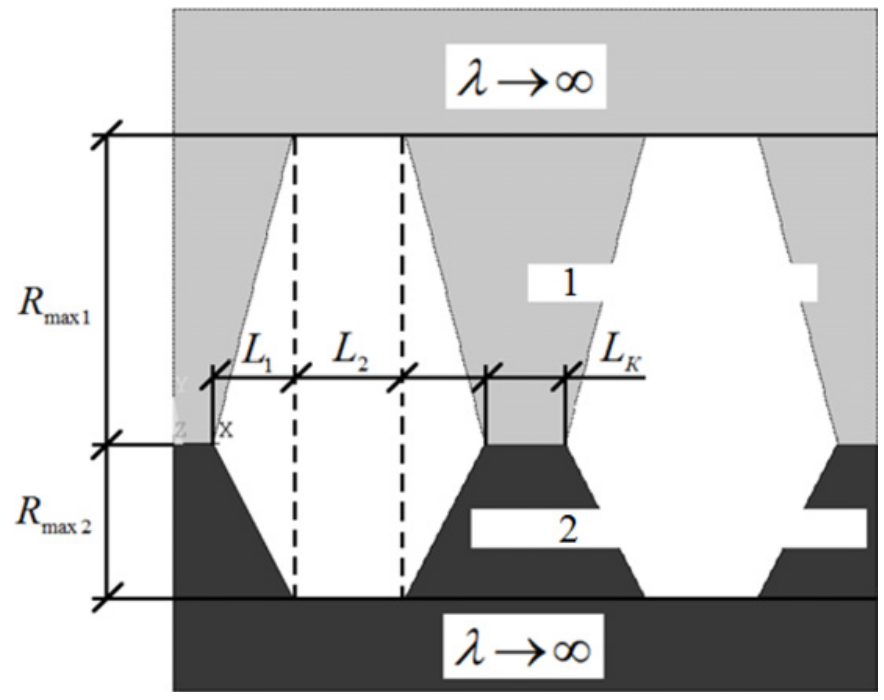

Figure 4. Simplified model geometry: 1 - nickel alloy; 2 - nitride ceramics. 


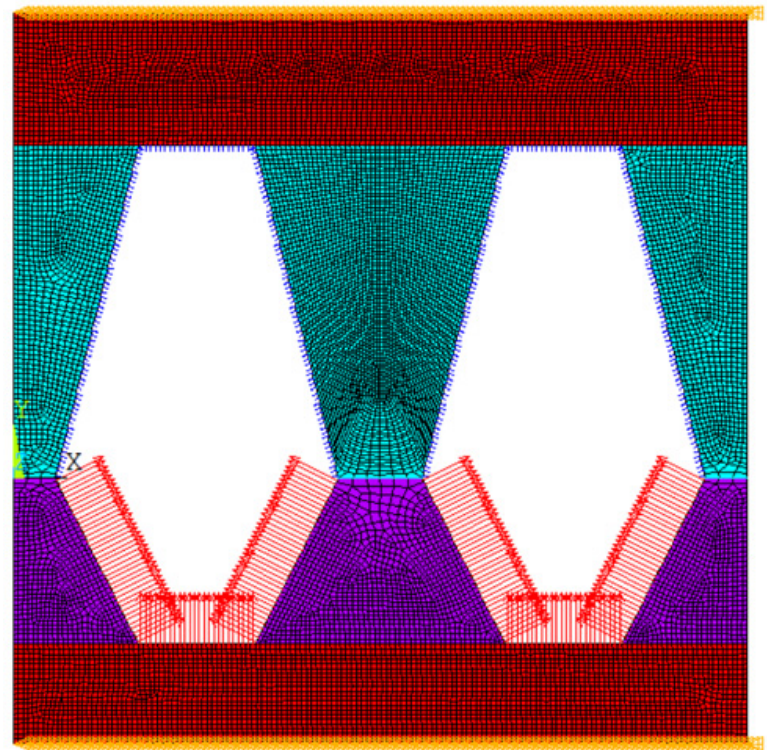

Figure 5. Finite element contact zone model.

Table 1. Simplified model parameters.

\begin{tabular}{|l|l|l|l|l|l|}
\hline$R_{\max 1}, \mu \mathrm{m}$ & $R_{\max 2}, \mu \mathrm{m}$ & $L_{k}, \mu \mathrm{m}$ & $L_{1}, \mu \mathrm{m}$ & $L_{2}, \mu \mathrm{m}$ & $\lambda_{l}, \mathrm{~W} / \mathrm{m} \cdot{ }^{\circ} \mathrm{C}$ \\
\hline 2,21 & 1,19 & 0,0485 & 0,43 & 0,01 & 3,91 \\
\hline
\end{tabular}

The ceramic material boundary subdomain from $x=0$ to $x=L$ in both models has been divided into 20 equal segments, the heat flux density $\left(q_{t}\right)$ has been determined from the relation:

$$
q_{t}=\frac{1}{L} \cdot \int_{0}^{L} q_{y}(x) d x
$$

Due to the areas smallness, the assumption of the flux density linear dependence on the $x$ coordinate on all segments has been taken:

$$
q_{y}=a \cdot x+b .
$$

Integral to the area $k-l$ :

$$
\int_{k}^{l} q_{y}(x) d x=\int_{k}^{l}(a \cdot x+b) d x=\frac{(l-k) \cdot(2 \cdot b+a \cdot k+a \cdot l)}{2} .
$$

Constants $a$ and $b$ have been calculated from the relations:

$$
\begin{gathered}
a=\frac{q_{y 2}-q_{y 1}}{x_{2}-x_{1}} ; \\
b=q_{y 1}-\frac{\left(q_{y 2}-q_{y 1}\right) \cdot x_{1}}{x_{2}-x_{1}} . \\
01034-\text { p. } 4
\end{gathered}
$$


Thermophysical Basis of Energy Technologies

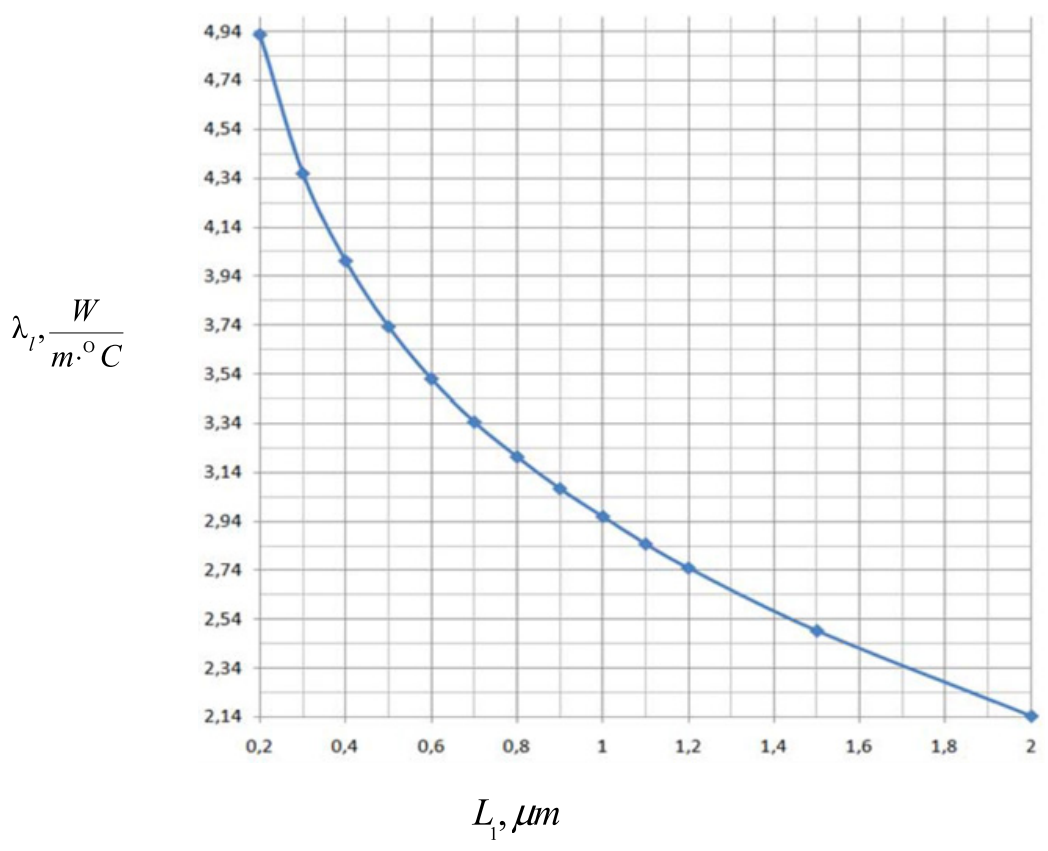

Figure 6. The influence of $L_{1}$ on the contact layer thermal conductivity coefficient.

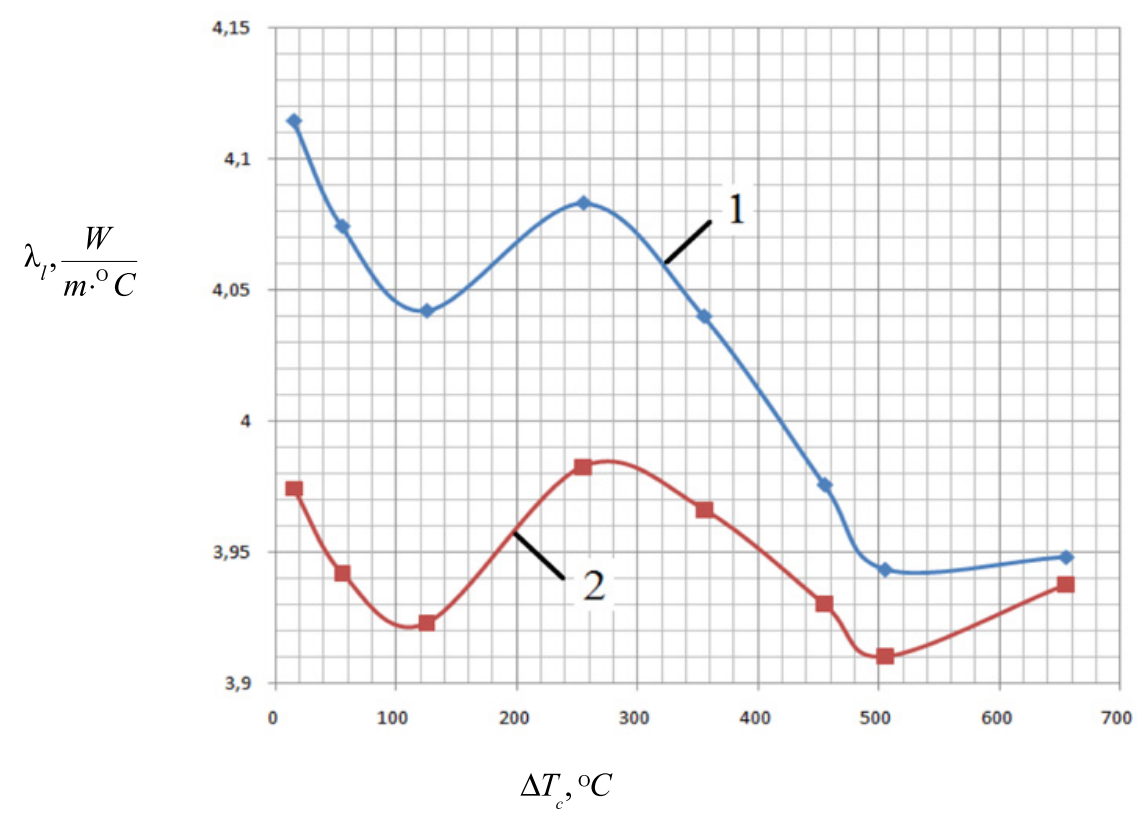

Figure 7. The comparison of contact layer thermal conductivity coefficients at $\Delta T_{c}=10^{\circ} \mathrm{C}: 1-$ the "real" model; 2 - the simplified model. 
Calculations have been carried out at the temperature drop of $\Delta T_{c}=10,30,50,70,90$ degrees respectively. Average temperature 20 variants in the contact zone $T_{a}$ have been considered for each $\Delta T_{c}$. Figure 2 shows the dependence of the contact layer $\lambda_{l}$ thermal conductivity coefficient on the value $T_{a}$. Figure 3 shows the dependence of the thermal conductivity coefficient average value on the temperature difference in the contact area.

The simplified roughness model has been also considered. The values of $R_{\max 1}=2.21 \mu \mathrm{m}, R_{\max 2}=$ $1.19 \mu \mathrm{m}$ (Figs. 4, 5) have been taken in the simplified nickel and ceramic parts thermal contact model.

The initial values have been chosen so to obtain the thermal conductivity coefficient, close to the "real" roughness model (at $T_{a}=505^{\circ} \mathrm{C}$ ), and considering the "real" roughness geometry similarity. In a simplified model (Table 1): $\frac{F_{f}}{F_{n}}=5,4 \% ; k_{p}=0,55$.

The influence of $L_{1}$ on the contact layer thermal conductivity coefficient can be observed by the results shown in Fig. 6.

A comparison of the results of the "real" and the simplified roughness model at $\Delta T_{c}=10^{\circ} \mathrm{C}$ have been carried out. As can be seen, the simplified model provides a very good similarity to a "real" roughness model (Fig. 7).

\section{Conclusion}

The technique of modeling has been developed, and with its help the thermal conductivity in the ceramic and metallic details contact zone in conditions, equivalent to gas turbines operation, has been investigated.

\section{References}

[1] V.A. Malkov, O.N. Phavorskii, V.N. Leontiev, Contact heat transfer in gas turbine engines and power plants (Mashinostroyenie, Moscow, 1978) [in Russian]

[2] S.V. Resnick, D.V. Sapronov, Design of ceramic blade and metal gas turbine disk tool joint (Izvestiya vuzov, Mashinostroyenie, 2014) [in Russian]

[3] I.A. Birger, B.F. Shorr, G.B. Iosilevich, Calculating the machine parts strength, (Mashinostroyenie, Russia, 1993) [in Russian] 